\title{
Aplicación móvil con Realidad Aumentada para localización de válvulas de agua potable
}

\section{Mobile application with Augmented Reality for drinking water valves location.}

\author{
S. Quevedo*, M. Zhindon y P. Mata, \\ Universidad Católica de Cuenca \\ Azogues, 030102, Ecuador \\ asquevedos@ucacue.edu.ec
}

\begin{abstract}
Resumen
La Empresa Pública de Agua Potable y Alcantarillado de Azogues (EMAPAL E.P.), registra pérdidas debido a fugas en la infraestructura del 58,46\% de su producción anual, esto en términos monetarios significa una pérdida de $\$ 740,076.48$ por año. A pesar de que se han desarrollado técnicas y procedimientos para minimizar las pérdidas y detectar fugas, este es un problema común en países en desarrollo. El propósito de este trabajo es implementar una aplicación móvil que utilice la Realidad Aumentada (RA) como técnica para capturar información primaria en la navegación, que permita localizar oportunamente las válvulas de aislamiento de la red de distribución para la reparación de daños que provocan las fugas. La aplicación se desarrolló con el framework libre y de código abierto Apache Cordova que combina el uso de tecnología web HTML, CSS, Javascript y nativas de la plataforma del dispositivo móvil. Como resultado se obtuvo una aplicación que captura datos del entorno de manera constante aumentando la información de la realidad con etiquetas georeferenciadas en la pantalla del dispositivo recuperadas desde la base de datos geográfica Spatialite-Android embebida en el dispositivo. El uso del Sistema Basado en Localización (SBL) con RA para la ubicación de válvulas evidenció una mejora en este procesos en un $32 \%$.
\end{abstract}

Palabras clave: realidad aumentada, navegación exploratoria, dispositivo móvil, aplicación móvil.

\begin{abstract}
The Potable Water and Sewerage Public Company of Azogues (EMAPAL E.P.) registers losses of 58.46\% on its annual production due to leaks on its infrastructure, which, in monetary terms, can be translated into a loss of \$740,076.48 per year. Although different techniques and procedures have been developed to minimize losses and to detect water leaks, this is a common problem in developing countries. The purpose of this work is to implement a mobile application using Augmented Reality (AR) as a technique for collecting primary information in navigation, so as to timely locate isolation valves of the distribution system, in order to repair the damages caused by water leakage. The application was developed with the Apache Cordova free and open source framework, which combines the HTML, CSS, Javascript and nativas web technologies of the mobile device's platform. As a result, an application that constantly captures information from the environment, augmenting the information of reality by using geo-referenced labels on the device's screen, was obtained. These labels are retrieved from the Spatialite-Android geographic database, embedded in the device. The use of location Based System (LBS) with RA for valves location, has evidenced a $32 \%$ improvement in this process.
\end{abstract}

Key words: augmented reality, exploratory navigation, mobile device, hybrid application.

\section{INTRODUCCIÓN}

$\mathrm{L}$ A Empresa Pública de Agua Potable y Alcantarillado de Azogues (EMAPAL E.P.), registra un total de 9990 usuarios con conexión domiciliaria de agua potable, y atiende al 57,34\% del total de predios del cantón, con una producción de 5,799,965.00 $\mathrm{m}^{3}$ de agua potable por año, con un promedio de pérdida de agua de 3,363,984.00 $\mathrm{m}^{3}$, es decir 58,46\% al año [1]. Entre varias de las causas de la pérdida se encuentra la detección tardía de las fugas de agua y el desgaste de tiempo en la búsqueda de las válvulas, que permiten aislar el daño para corregir el problema, esto debido a que la localización de los accesorios se realiza de forma empírica.

Las pérdidas de agua son un problema frecuente sobre todo en países en desarrollo. Se ha demostrado que a través de la investigación, países desarrollados han podido reducir en gran medida este problema. En este contexto, entre otras, una manera efectiva de minimizar este problema, es mediante la reparación de fugas de forma oportuna [2].

Las pérdidas en la ciudad de Azogues desde un punto de 
vista financiero representan una pérdida comercial promedio de $\$ 0,22$ por cada $m^{3}$ producido, es decir, una pérdida monetaria anual de $\$ 740,076.48$ [3].

En un escenario común cuando se detecta un falla, generalmente un técnico de operación y mantenimiento de la EMAPAL E.P., emplea la localización empírica de los accesorios de la red de distribución, mediante un proceso de prueba y error, por esta razón el operario pierde tiempo recorriendo la red hasta la válvula correcta.

Un sistema de abastecimiento de agua es el encargado de garantizar su transporte desde las fuentes de captación hasta las acometidas para su suministro a los usuarios finales. Para que esto se lleve a cabo, el sistema debe garantizar los siguientes controles: del agua y sus condiciones de suministro, de pérdidas, y de costes de mantenimiento[4].

Con respecto al control de las pérdidas, el problema en este se produce entre otros, debido a circunstancias como la fragilidad institucional e infraestructuras deficientes existentes. Es necesario diferenciar que las pérdidas pueden ser físicas o monetarias, en el primer caso cuando el recurso no llega a satisfacer las necesidades de un usuario, mientras que en el segundo, desde el punto de vista de la empresa, se produce cuando el agua que se extrae de sus fuentes naturales no se traduce en ingreso económico para la empresa, es decir, no recibe el pago por el recurso, ya sea por fugas, errores en los cobros o rubros de agua [2].

Para reducir estas pérdidas debido a deficiencias en la infraestructura, existen técnicas para la detección de fugas como las que se detallan a continuación: de balance de agua, medición en distritos hidrométricos, por sonido, por presión diferencial, por trazadores [5].

Además de las técnicas para la detección de fugas, existen procedimientos para su localización, a través de los cuales es posible identificar el lugar en la red de distribución en donde se produce la pérdida. Ya que gran parte de las pérdidas se producen debido a fallos por defectos en la infraestructura también existen estrategias para su localización que dependen de: su ámbito de aplicación, rango de detección, eficacia del método y eficiencia volumétrica [4].

Una vez que se ha diferenciado los tipos de pérdidas y conociendo que existen técnicas para la detección y procedimientos para la localización de fugas, estas fuentes no tratan como localizar las válvulas para realizar el aislamiento de las fugas, para proceder con su reparación. Si bien es cierto, en la técnica de detección por sectores hídricos, se menciona, que es necesario disponer de un inventario de red de distribución, no se hace énfasis, en cómo utilizar este inventario para la detección de los componentes de la red.

Desde el punto de vista de la ciencia de la información geográfica, la detección de las válvulas puede ser visto como un problema de navegación en búsqueda de un objeto en en el espacio geográfico.

La navegación es el desplazamiento en o alrededor de un entorno, puede ser exploratoria o de búsqueda de rutas o destinos específicos. La información del espacio se obtiene de fuentes primarias y secundarias [6]. Las fuentes primarias constituyen información que puede ser captada con nuestros sentidos desde el entorno en el cual nos encontramos inmersos [7], por ejemplo todos los elementos que podemos captar con nuestros ojos nos proveen de conocimiento del espacio en el que nos encontramos. Por otra parte, una fuente secundaria es información que se puede obtener sin inmersión en el entorno, por ejemplo consultando un mapa de lugares a visitar mientras nos encontramos en un hotel [6].

Los sistemas de navegación tradicionales presentan la información del entorno en forma de gráficos abstractos como mapas impresos o digitales [8], por esta razón el usuario tiene que fijarse en su entorno constantemente para entender la información que el mapa representa [7].

Un SBL proporciona información mediante un dispositivo que es capaz de conocer su ubicación geográfica y modificar la información proporcionada conforme cambia su ubicación [7]. Los sistemas de navegación tradicionales son SBL que presentan la información en mapas digitales de calles y puntos de interés (POI). Los POI son lugares conocidos que se encuentra en el entorno del usuario [9]. OSMAND es una aplicación LBS para dispositivos móviles que presenta información de POIs y callejeros en función de la posición geográfica del dispositivo.

El desarrollo de la siguiente generación de SBL tienden al uso de la RA [10]. Según [11] la RA combina la realidad con objetos virtuales en tiempo real. Los objetos virtuales son contenidos digitales generados por computadora para aumentar la información acerca de los elementos del entorno real [12].

La RA detecta objetos específicos del entorno real mediante el reconocimiento de marcadores e imágenes a través de la cámara, o por su ubicación geográfica mediante el uso del GPS [13], presentando anotaciones georeferenciadas sobre los objetos reales del entorno capturados mediante la cámara del dispositivo, por lo tanto la RA se puede utilizar como técnica para capturar información primaria y aumentar la percepción del usuario mostrando información adicional de los elementos del entorno [6], permitiendo capturar información primaria de manera constante a través del dispositivo y aumentar esta información con contenido digital presentado en la pantalla del dispositivo [8].

El estándar de lenguaje de marcado para realidad aumentada ARML 2.0 $0^{1}$ fue desarrollando por el consorcio geoespacial abierto $\mathrm{OGC}^{2}$, y concebido como un formato para describir los objetos virtuales y su posicionamiento sobre los objetos de la realidad. El estándar además determina que es necesario que el dispositivo en donde se desea implementar una escena de RA debe contar con sensores de orientación, GPS y cámara [14].

Las capacidades de procesamiento que poseen en la actualidad los dispositivos móviles y los componentes que portan como cámara, acelerómetro y GPS, hacen que sea posible el desarrollo de aplicaciones RA basadas en locali-

\footnotetext{
${ }^{1}$ Augmented Reality Markup Language 2.0

${ }^{2}$ Open Geospatial Consortium
} 
zación [15].

El objetivo de este trabajo es implementar un prototipo de navegación con RA utilizando estándares para la creación de una aplicación móvil que capture información primaria y aumente la percepción del entorno durante la navegación, mejorando las capacidades sensoriales biológicas del usuario con contenido sintético generado por computador para que este pueda entender de mejor manera su entorno y que permita optimizar la localización de las válvulas de aislamiento para la reparación de las fugas en la infraestructura de distribución del agua potable.

\section{Metodología}

El enfoque de este trabajo es la creación de un prototipo de aplicación móvil con RA, que permita la localización geográfica, rutas de llegada y descripción de válvulas que se encuentran situados en una red de distribución de agua potable. En el contexto de este trabajo se toma como referencia la empresa de agua potable de la ciudad de Azogues EMAPAL E.P.

Del personal que labora en la empresa EMAPAL E.P., los únicos con la capacidad de operar y manipular las redes de agua potable son los trabajadores de los departamentos técnico y de planificación; dando un total de 6 los encargados de esta labor.

Para realizar las pruebas del proceso de identificación de válvulas se crea una ficha de observación (ver Tabla I), que recoge el número de intentos que le toma a cada técnico en identificar dos válvulas aleatorias por medio de un proceso manual y dos válvulas adicionales con el proceso de RA.

TABLA I

FICHA PARA TOMAR RESULTADOS DE INTENTOS. Fuente: Autor

\begin{tabular}{|l|l|l|l|l|}
\hline & \multicolumn{3}{|c|}{ INTENTO PARA ENCONTRAR VÁLVULA } \\
\hline & \multicolumn{2}{|c|}{ MANUAL } & REALIDAD AUMENTADA \\
\hline & VALVULA 1 & VALVULA 2 & VALVULA 3 & VALVULA 4 \\
\hline Técnico 1 & & & & \\
\hline Técnico 2 & & & & \\
\hline Técnico 3 & & & & \\
\hline Técnico 4 & & & & \\
\hline Técnico 5 & & & & \\
\hline Técnico 6 & & & \\
\hline
\end{tabular}

\section{A. Arquitectura del prototipo}

La arquitectura propuesta en este trabajo tiene la intención de mostrar en un dispositivo móvil un proceso de realidad aumentada para la localización geográfica, rutas de llegada y descripción de válvulas que se encuentran situados en una red de distribución de agua potable, tomando como base la información cartográfica generada por las empresas que distribuyen el líquido vital. La Figura 1 muestra la arquitectura prevista y a continuación se describe el proceso a seguir:

1) Migración de datos: El objetivo principal de este paso, es la migración de los datos geográficos correspondientes a las válvulas de agua, hacia una base de datos que

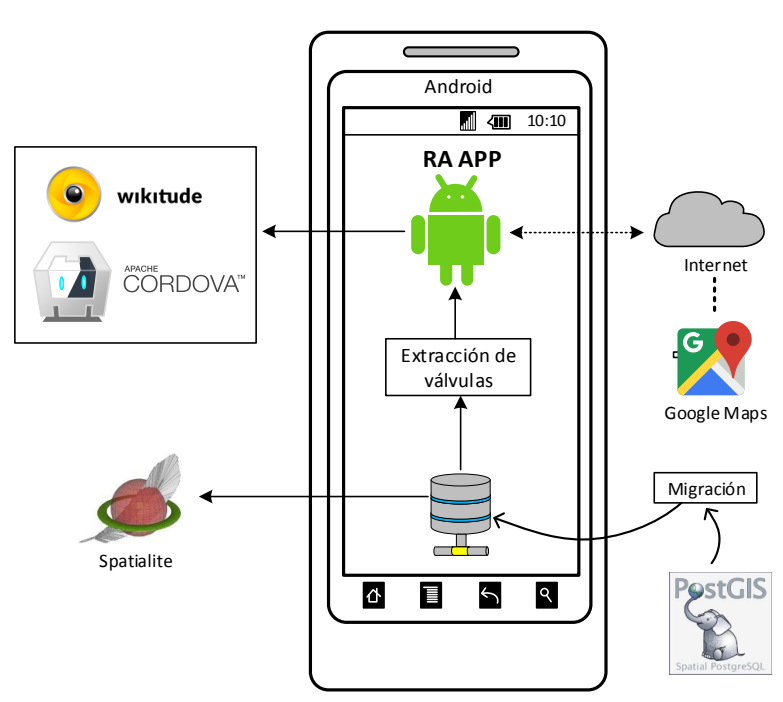

FIG. 1. Arquitectura de la aplicación. Fuente: Autor

tenga soporte geográfico y que opere en un dispositivo móvil. El proceso de migración sin importar la fuente de los datos ya sea un archivos plano o en una base de datos geográfica, se ejecuta usando dos herramientas SIG, La herramienta QGIS $^{3}$ para la carga, procesamiento y migración de los datos y la base de datos SpatiaLite $^{4}$, que almacena los datos geográficos y que puede ser transferida a un sistema operativo móvil para su uso. En la empresa EMAPAL E.P.; la migración se realiza desde una base datos Postgis ${ }^{5}$; hacia la base de datos Spatialite, utilizando el sistema de información geográfico QGIS con su complemento qspatialite ${ }^{6}$. Los accesorios contienen datos geográficos de latitud, longitud, altura y una descripción alfanumérica de los accesorios.

2) Extracción de válvulas: El objetivo de este proceso es la extracción de información de las válvulas considerando lo siguiente: Seleccionar la descripción, latitud, longitud y la distancia desde el dispositivo hasta la válvula; finalmente ordenar los datos por la distancia de menor a mayor. La Figura 2, muestra el código SQL utilizado para obtener los datos.

3) Realidad aumentada: Para el proceso de RA es necesario crear una aplicación móvil que permita la navegación en entornos preparados, para la ejecución de estas actividades se han usado las siguientes herramientas: Apache Cordova como framework para el desarrollo de la aplicación; SDK Wikitude para capturar y aumentar la información primaria del entorno por último el pluguin Android-Spatialite para el acceso a la base de datos geográfica.

\footnotetext{
${ }^{3}$ http://www.qgis.org

${ }^{4}$ https://www.gaia-gis.it/fossil/libspatialite/index

${ }^{5}$ http://postgis.net/

${ }^{6} \mathrm{https} / / /$ plugins.qgis.org/plugins/QspatiaLite/
} 


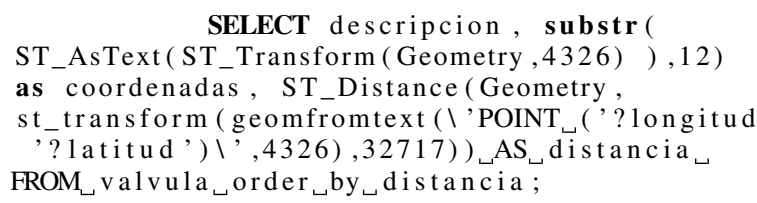

FIG. 2. SQL para extracción de válvulas. Fuente: Autor

4) Rutas de llegada. El cálculo de la mejor ruta desde el punto de ubicación del dispositivo, hasta la válvula seleccionada por el usuario; se realiza con la integración del API de rutas de google, para esto se construye un enlace web apuntando a los servidores de google que incluye la longitud y latitud del punto de partida y el punto de llegada.

\section{B. Inicialización}

En esta etapa se determinan las herramientas más importantes y arquitectura empleadas para la implementación del prototipo.

- Apache Cordova es un framework libre y de código abierto que permite el desarrollo multiplataforma utilizando tecnologías web como HTML, CSS Y Javascript embebidas en una aplicación nativa. Las aplicaciones híbridas se desarrollan mediante una combinación de tecnologías web y nativas de la plataforma del dispositivo móvil.[16].

- La plataforma Android es un conjunto de librerías, binarios y herramientas, incluyen el SDK para el desarrollo de aplicaciones Android[17].

- Spatialite es un sistema gestor de base de datos espacial que implementa el estándar OGC-SFS acoplado en la base de datos SQLite. El motor de la base de datos SQLite se basa en el estándar SQL92. [18].

- Wikitude SDK es el nucleo para el desarrollo de la aplicación con RA. Esta herramienta reduce la complejidad de la implementación de aplicaciones con RA para dispositivos móviles [19].

\section{RESULTADOS}

En esta sección se describe los resultados de la creación de la aplicación con realidad aumentada y se realiza un análisis de la eficiencia del mismo frente al proceso manual.

\section{A. Aplicación RA}

La aplicación RA cuenta con diversas prestaciones que permiten una mejor interacción con el usuario (Figura 3), tales como: la aumentación de las válvulas, la distancia y la descripción de la válvula, además cuenta con una brújula que sirve como instrumento de orientación.

Una vez elegida la válvula de destino, la aplicación RA permite hacer un cálculo de ruta descriptiva y gráfica, como muestra la Figura 4.

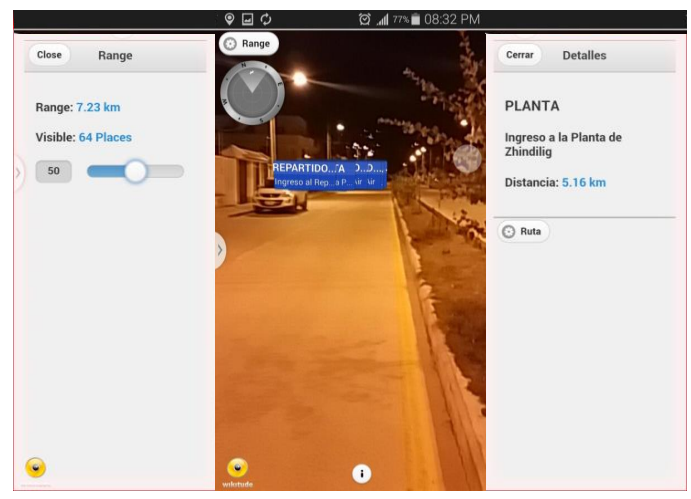

FIG. 3. Aplicación RA. Fuente: Autor

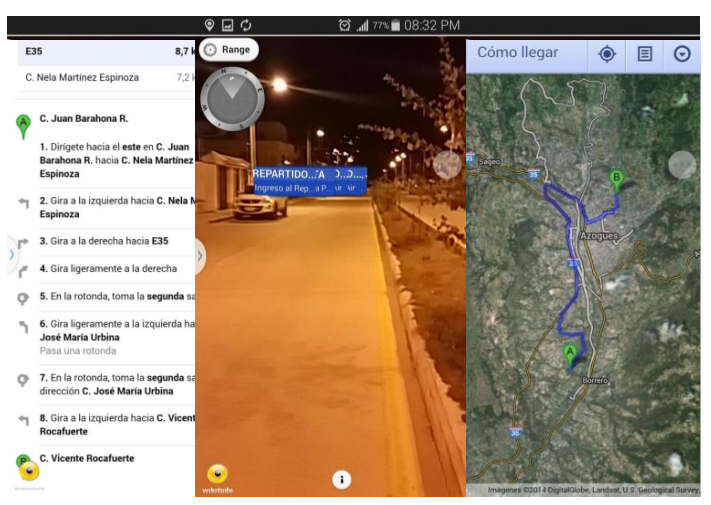

FIG. 4. Aplicación RA - Ruta. Fuente: Autor

\section{B. Eficiencia}

Para el análisis de la eficiencia, se realizan las pruebas respectivas, llenando la Ficha I con los resultados que muestran la Tabla. III, para ello 6 técnicos de la EMAPAL E.P. proceden a la búsqueda empírica de 2 válvulas, al cual le llamamos proceso manual, seguido una búsqueda de 2 válvulas a través de la Aplicación $R A$.

En el contexto de estos resultados, se muestra al Técnico 1 que en el proceso manual realiza 5 y 4 intentos para identificar las válvulas 1 y 2, respectivamente; mientras que con la aplicación RA, se obtiene al primer intento en el hallazgo de las válvulas 3 y 4 . Para ver los datos de una mejor manera se construye un gráfico de araña (Figura 5), que indica qué proceso de localización muestra un mejor desempeño.

La Tabla III muestra los resultados consolidados y que se encuentran organizados por el número de intentos, tanto con el proceso manual como con RA. Así por ejemplo, se muestra en la primera fila que, 8 búsquedas fueron efectivas al primer intento con el proceso manual y 9 veces con RA. Considerando que el ideal de búsquedas al primer intento es de 12, debido a que son 6 técnicos que localizan 2 válvulas. 
TABLA II

RESUlTADOS DE INTENTOS. Fuente: Autor

\begin{tabular}{|l|c|c|c|c|}
\hline & \multicolumn{3}{|c|}{ INTENTOS PARA ENCONTRAR VÁLVULA } \\
\hline & \multicolumn{2}{|c|}{ MANUAL } & \multicolumn{2}{c|}{ REALIDAD AUMENTADA } \\
\hline & VALVULA 1 & VALVULA 2 & VALVULA 3 & VALVULA 4 \\
\hline Técnico 1 & 5 & 4 & 1 & 1 \\
\hline Técnico 2 & 1 & 1 & 1 & 1 \\
\hline Técnico 3 & 1 & 1 & 1 & 1 \\
\hline Técnico 4 & 3 & 1 & 1 & 2 \\
\hline Técnico 5 & 1 & 1 & 1 & 1 \\
\hline Técnico 6 & 5 & 1 & 2 & 2 \\
\hline
\end{tabular}

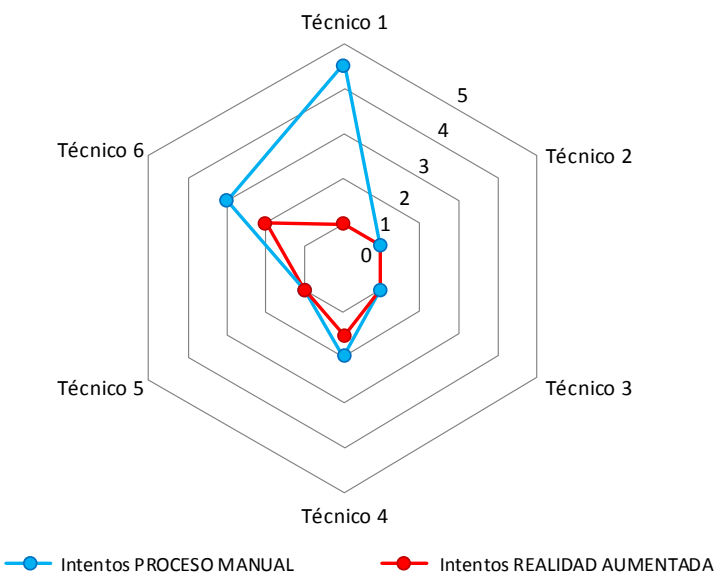

FIG. 5. Resultados de intentos. Fuente: Autor

Gráficamente se puede observar en la Figura 6 que la eficiencia de localización de válvulas mediante la RA es significativamente mayor que hacerlo a través del proceso manual.

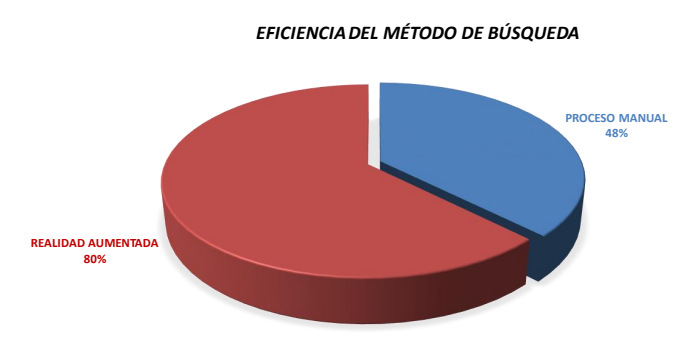

FIG. 6. Eficiencia del sistema. Fuente: Autor

\section{DISCUSIÓN}

El prototipo implementado contribuye a la consecución del objetivo principal que es el desarrollar una aplicación que utilice la técnica de la realidad aumentada para capturar información primaria durante la navegación exploratoria.

Durante el desarrollo del prototipo resultó complejo integrar todas las herramientas a utilizar y conseguir su fun-
TABLA III

PRoces miento DE RESUlTAdos. Fuente: Autor

\begin{tabular}{cccccc} 
INTENTOS & MANUAL & RA & TOT. MANUAL & TOT. RA & IDEAL \\
\hline 1 & 8 & 9 & 8 & 9 & 12 \\
2 & 0 & 3 & 0 & 6 & 0 \\
3 & 1 & 0 & 3 & 0 & 0 \\
4 & 1 & 0 & 4 & 0 & 0 \\
5 & 2 & 0 & 10 & 0 & 0 \\
\hline TOTAL & & & 25 & 15 & 12 \\
\hline PORCENTAJE & & & $48 \%$ & $80 \%$ & $100 \%$
\end{tabular}

cionalidad. La implementación de la realidad aumentada con Wikitude fue relativamente sencilla como lo expresa [19]

Contrariamente a que la aplicación se encuentra desarrollada con el framework Apache Cordova, que permite el desarrollo de aplicaciones híbridas multiplataforma[16], el uso de la base de datos geográfica Spatialite-android limita su uso a dispositivos con sistema operativo Android.

Cuando se recupera una gran cantidad de POIs y se presentan en la pantalla mediante etiquetas, estos saturan la pantalla del dispositivo. Por esta razón fue necesario limitar la consulta SQL a las 25 válvulas más cercana al dispositivo. Wikitude además provee de una herramienta adicional que permite reducir la cantidad de POIs que se muestran en la pantalla en función de su distancia relativa a la ubicación del dispositivo.

El funcionamiento del prototipo se encuentra limitado también por la precisión del GPS, cuando la aplicación realiza la aumentación de los POIs que se encuentran a más o menos cinco metros de distancia de la ubicación actual del dispositivo, los marcadores empiezan a cambiar su posición en la pantalla de forma arbitraria.

Concluida la implementación el prototipo, este evidencia que la realidad aumentada puede ser utilizada como técnica en el desarrollo de aplicaciones LBS como lo afirma [10], permite capturar la información del entorno de manera constante y aumenta la comprensión del mismo presentando en la pantalla del dispositivo información adicional recuperada de la base de datos en forma de etiquetas georeferenciadas de manera combinada con la realidad capturada por la cámara del dispositivo demostrando lo planteado por $[13,6,6]$.

\section{CONCLUSIONES Y RECOMENDACIONES}

De acuerdo a los datos obtenidos y procesados se puede identificar que el proceso de localización con RA es un $32 \%$ más eficiente que el proceso manual, estos datos pueden variar si se aplica un mayor factor de confianza para la determinación de la muestra al momento de recoger la información, puesto que este trabajo se limitó a una muestra muy pequeña por falta de disponibilidad de los técnicos de la EMAPAL E.P. Se recomienda repetir el proceso de localización de válvulas descrito anteriormente con un intervalo de confianza de al menos el $90 \%$, para obtener porcentajes de eficiencia definitivos. 
El prototipo implementado sugiere que la RA puede ser empleada como técnica para la visualización y aumentación de información del entorno en sistemas de navegación LBS. El uso de la RA en LBS no descarta las técnicas tradicionales como presentación de información en mapas, al contrario se sugiere combinar las dos técnicas para analizar si de manera conjunta mejoran la percepción del entorno y permiten una navegación más fluida e intuitiva. Además se sugiere combinar las técnicas de detección de objetos del entorno real a través del reconocimiento de marcadores o imágenes conjuntamente con el reconocimiento por localización geográfica para mejorar la precisión con la que se presentan los marcadores con la información aumentada recuperada de la base de datos sobre los objetos reales, con el fin de minimizar la limitante por la precisión del GPS. No existe una tecnología propia para el desarrollo de RA, al contrario se utiliza un conjunto de herramientas tecnológicas existentes para conseguir una percepción visual [20], por esta razón se puede referir a la RA como una técnica y no como una tecnología.

\section{REFERENCIAS}

[1] EMAPAL, “Acerca de,” tech. rep., Empresa Municipal de Agua Potable y Alcantarillado del Cantón Azogues, 2014.

[2] Instituto Mexicano de Tecnología del Agua, "Conceptos de reducción y control de pérdidas, y de sectorización de redes de distribución," 2007.

[3] EMAPAL, "Informe de gestión,” tech. rep., Empresa Municipal de Agua Potable y Alcantarillado del cantón Azogues (EMAPAL), 2014.

[4] A. Vela, F. Martínez, J. García-Serra, and R. Pérez, "Estrategias óptimas para la reducción de pérdidas de agua en sistemas de abastecimiento," Ingeniería del agua, vol. 1, no. 1, 1994.

[5] L. Ochoa Alejo and V. Bourguett Ortiz, "Reducción integral de pérdidas de agua potable," 2001.

[6] R. Grasset, A. Mulloni, M. Billinghurst, and D. Schmalstieg, "Navigation techniques in augmented and mixed reality: crossing the virtuality continuum," in Handbook of Augmented Reality, pp. 379-407, Springer, 2011.

[7] P. A. Longley, M. F. Goodchild, D. J. Maguire, and D. W. Rhind, Geographic information science and systems. John Wiley \& Sons, 2015.

[8] T. Jackson, F. Angermann, and P. Meier, "Survey of use cases for mobile augmented reality browsers," in Handbook of Augmented Reality, pp. 409-431, Springer, 2011.

[9] J. M. Luna, R. Hervás, J. Fontecha, and J. Bravo, “A friendly navigation-system based on points of interest, augmented reality and context-awareness," in Ubiquitous Computing and Ambient Intelligence, pp. 137144, Springer, 2012.

[10] G. Percivall, "Increasing market opportunities for augmented reality through collaborative development of open standards," in A Position Paper for the International AR Standards Meeting, 2011.

[11] R. T. Azuma, "A survey of augmented reality," Presence: Teleoperators and virtual environments, vol. 6, no. 4, pp. 355-385, 1997.

[12] M. Prenda and S. Valente, "Mpeg vision, position and technologies for augmented reality," July 2011.

[13] J. Jeon, S. Kim, and S. Lee, "Considerations of generic framework for ar on the web," in Proceedings of W3C Workshop: Augmented Reality on the Web, Spain, 2010.

[14] M. Lechner, "Ogc augmented reality markup language 2.0 (arml 2.0)," 2015.

[15] R. Paucher and M. Turk, "Location-based augmented reality on mobile phones," in Computer Vision and Pattern Recognition Workshops (CVPRW), 2010 IEEE Computer Society Conference on, pp. 9-16, IEEE, 2010.

[16] J. Wargo, Apache Cordova 3 programming. Mobile Programming Series, Addison-Wesley Professional, 2013.

[17] F. Ableson, R. Sen, C. King, and C. E. Ortiz, Android in Action. Greenwich, CT, USA: Manning Publications Co., 2011.

[18] A. Furieri, "Spatialite cookbook," Fine Dining Experience: Chez Dijkstra, vol. 151, 2011.

[19] L. Madden, Professional augmented reality browsers for smartphones: programming for junaio, layar and wikitude. John Wiley \& Sons, 2011.

[20] G. Kipper and J. Rampolla, Augmented Reality: an emerging technologies guide to AR. Elsevier, 2012.

Recibido: 15 de junio de 2017

Aceptado: 30 de agosto de 2017

Sebastian Quevedo: Ingeniero de Sistemas, docente en la Facultad de Tecnologías de la Información y Comunicación de la Universidad Católica de Cuenca.

Martin Zhindon: Cuenta con experiencia en la implementación de sistemas de información empresariales y geográficos. Interesado en la ciencia y sistemas de información geográfico en cuidados de la salud.Correo electrónico: mgzhindonm@ucacue.edu.ec

Paul Mata: Ingeniero Electrónico, docente en la Faculdad de Ingeniería, Construcción e Industria de la Universidad Católica de Cuenca. Correo electrónico: jpmataq@ucacue.edu.ec 